\title{
Impact on the Volatility of the Bitcoin Price with the Implementation of Macro-Regulation
}

\author{
Siyao $\mathrm{Ma}^{1 *}$ \\ ${ }^{1}$ School of Mathematics and Statistics, The University of Sheffield, Sheffield, S10 2TN, United Kingdom \\ *Corresponding author. Email: sma25@sheffield.ac.uk
}

\begin{abstract}
After year two thousand, with the booming of internet networks, virtual currency has appeared into the public's view. In the midst of all (including 8861 numbers of virtual currency, calculate by Coinmarketcap), bitcoin has become the most represented currency for us to discuss. This paper returns the closed adjusted price calculates rates. ARMA models are used to test whether the time series is stationary or not. Dummy variables are set according to the time by specific policies are implemented. According to the GARCH model we used, the implementation of the policy does lead to a sharp decline in the price of bitcoin. Accordingly, in the development process of bitcoin, supervision does give a positive effect in maintaining the price to be balanced and a positive control on malicious speculation to monopolize the bitcoin market and blind purchasing.
\end{abstract}

Keywords: bitcoin price, ARMA model, dummy variables, GARCH model, correlation.

\section{INTRODUCTION}

Indeed, bitcoin is more like a medium of exchange, a "commodity"; since there is no specific exchange rate between bitcoin to any other currencies, it is hard to define its virtual value. Furthermore, bitcoin does not "store money". The price per unit is random, and no limited boundaries on it. In addition, some scholars do believe that bitcoins do satisfy some functions of virtual currency, but due to the difficulties of estimation its virtual value, and the limitation of the numbers of supply, brings that bitcoin is not firm enough to become a legal currency between countries or in the world; as a result, bitcoin is more likely to trade as an investment asset.

Amazingly, a huge risk of investment in bitcoin does not bring a more consideration transaction of purchasing for the investors. For decades, the price of one bitcoin has gone higher and higher, even got 64,863 USD at the vertex on April 14, 2021. The bitcoin price stands out among most virtual currencies because its total quantity is very limited maximum supply of $21,000,000$, the limit is hard-coded into the Bitcoin protocol and cannot be changed) and has strong scarcity [1]. Political decisions will not inflate this fixed supply.

There is evidence showing that financial assets can usually be affected by both market factors and policy factors. Some studies have found out that a huge price fluctuation may present after certain policy changes. In the United States, Bitcoin Suffers Steepest Drop in 10 Days as US Monetary Policy is published; and it continued its descent amid whispers of US Federal Reserve tapering economic stimulus and China's ongoing pressure on crypto miners [2].

Getting bitcoin in a mining system refers to carrying out mathematical operations for a bitcoin network through computer hardware, especially in China. Miners who provide services in this process can get a reward. Since the number of tokens issued is calculated according to the tasks completed by the miners, the mining competition is very fierce. The massive use of electricity follows this. Furthermore, more and more bitcoin miners seek cheaper power areas, for example, China; which means more miners might join in China. Hence the policy is quite necessary, not only aiming to prevent a balance control on the price of bitcoin but also takes a great consideration on the environmental governance and protection of those natural resources.

After more than ten years of development of bitcoin, the market has gradually improved. Due to the research started, the policy has been implemented for nearly three months. It is too early to give a conclusion for the effect of the Intervention of macro supervision. Hence, six months on the price of bitcoin will be included in 
this experiment (which starts three months before the policy, from February 18, 2021, to August 18, 2021). This study has a certain practical impact on the development of a special currency market.

\section{DATA AND METHODS}

\subsection{Data}

\subsubsection{Choosing Data}

To do this research, we started with finding the price of bitcoin [3], and the range of time setting is quite important. In order to restrain speculations and prevent sharp fluctuations in price, relevant policies have been issued in China in the way of Macro supervision. China Internet Finance Association and other two organizations have jointly issued the "Notice on prevention of bitcoin risk" and "Announcement on preventing the financing risk of token issuance" on May 18, 2021 [4]. Hence, the total time period will take place from the start of February 18, 2021, to August 19, 2021; and we take one day as a base unit. Adjusted closing price [5] of bitcoin has been taken in this measurement; it amends a stock's closing price to reflect that stock's value after accounting for any corporate actions, which is often seen when we examine historical returns that is the second step followed by.

Here is a time series plot drawn by $\mathrm{R}$ studio is shown below to give a brief look at the moving trend of the bitcoin price.

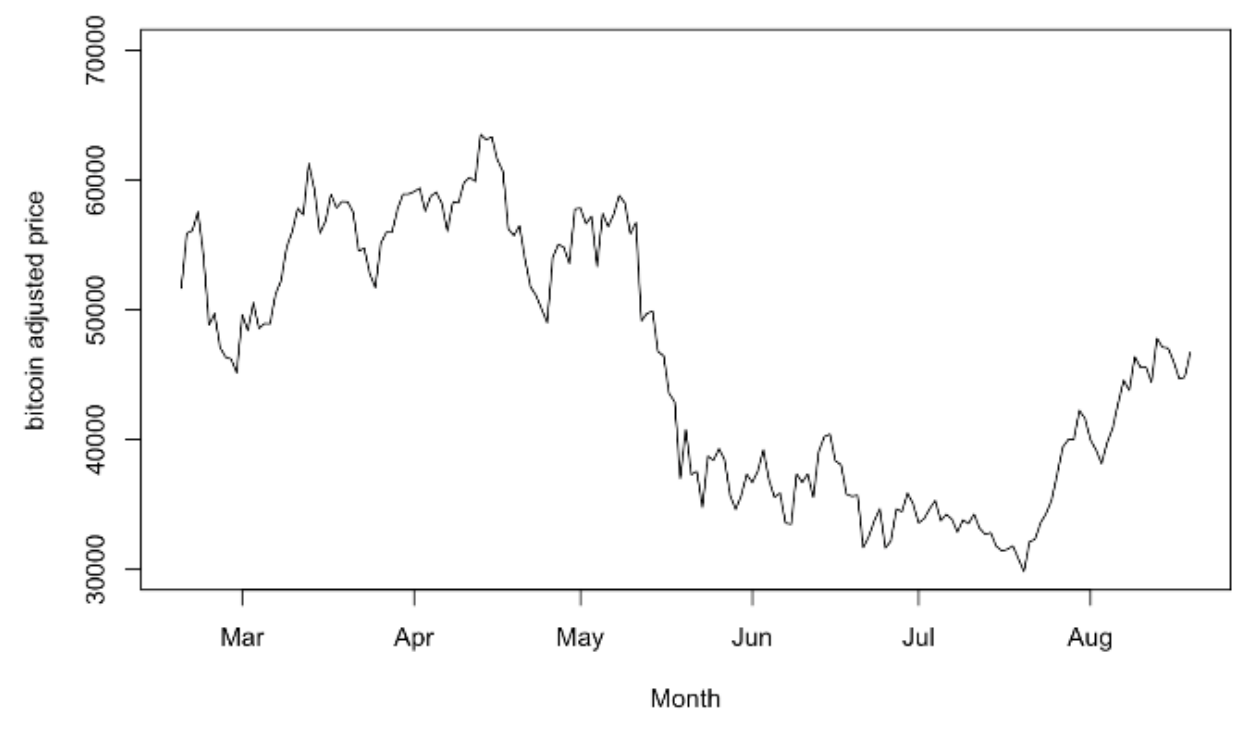

Figure 1. The time series plot of the bitcoin adjusted closing price with daily based.

\subsubsection{Logarithm Returns Rate of Bitcoin}

The reason for using a logarithm returns rate rather than just the adjusted price is that it measures all the data in a comparable metric. Hence, it enables us to evaluate and analyze relationships among variables despite originating them from price series of unequal values [6].

In order to find the logarithm returns rate, we need to calculate the returns rate first with the use of the following equation.

$$
\text { DailyRaturnsRate }=r=\frac{\left(P_{1}-P_{0}\right)}{P_{0}}
$$

In this equation $P_{1}$ stands for the adjusted closing price for today and $P_{0}$ stands for the adjusted closing price of yesterday, and we use $r$ to stand for the daily returns rate.

After that, the logarithm returns rate can be written down in the form shown below.

$$
\text { LogarithmRaturnsRate }=\ln (1+r)
$$

A time series plot drawn by $\mathrm{R}$ studio is shown below to give a brief look at the moving trend. In this graph, $\mathrm{n}$ stands for the number odder of the data, and there are 184 numbers of them in total. 


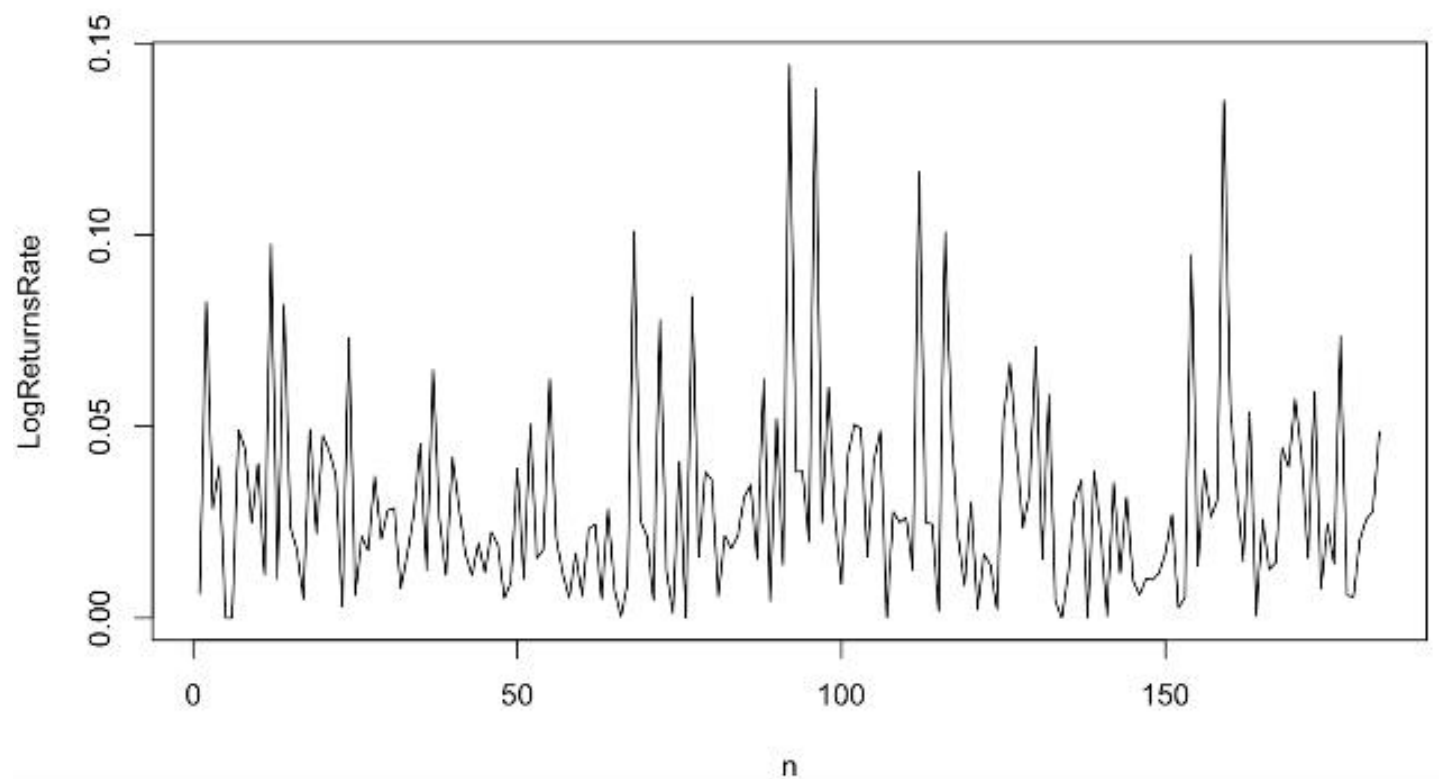

Figure 2. The time series plot of the logarithm returns rate.

\subsubsection{Testing of Stability and Stationarity of the Asset}

The reason for testing the stationarity of the asset is to decide which model we are using to analyze.

If stability conditions hold, stationarity conditions are satisfied (the converse is not true) and (stochastic process) is weakly stationary.

We can use R to simply check that the asset we are using in this experiment is weakly stationary; hence the next step is to decide which model we are using; is it 'AR' or 'MA' or ARMA.

\subsection{Methodology}

\subsubsection{Using of Autoregressive Moving Average (ARMA) Model}

An ARMA model is used to describe a weakly stationary stochastic time series in terms of two polynomials. It is often written in the way of the $\operatorname{ARMA}(p, q)$ model; in this case, $\mathrm{p}$ represents 'AR' in ARMA, which is the order of autoregressive polynomials; and q represents 'MA' which is the order moving average polynomials.

\subsubsection{Formula of ARMA Model}

First, talking about 'AR' tells us the relationship between the current value and the previous value, in order to predict the future value based on all the historical values. And the formula that we are using is written down in the form shown below.

$$
y_{t}=\mu+\sum_{i=1}^{p} \phi_{i} y_{t-i}+\epsilon_{t}
$$

In this equation, $y_{t}$ is the current value; $\mu$ is a constant; order $p$ is the lag value; $\phi_{i}$ is the autocorrelation coefficient; and $\epsilon_{t}$ is considered as the white noise.

For 'MA', it will help with canceling down the stochastic volatility. And the formula that we are using is written down in the form shown below.

$$
y_{t}=\mu+\sum_{j=1}^{q} \theta_{j} \epsilon_{t-j}+\epsilon_{t}
$$

In this equation, order $q$ is the lag value.

Hence, if we put 'AR' and 'MA' together, we may get the formula looking like this shown below.

$$
y_{t}=\mu+\sum_{i=1}^{p} \phi_{i} y_{t-i}+\sum_{j=1}^{q} \theta_{j} \epsilon_{t-j}+\epsilon_{t}
$$

\subsubsection{Formula of Auto-Correlation Function $(A C F)$ and Partial Auto-Correlation Function $(P A C F)$}

ACF gives us values of the Auto-Correlation with its lagged value, and it is plotted with the confidence band. In order to find out how the present value is related to its past values in a series, an ACF plot would give great help. An ACF plot is demonstrated below 


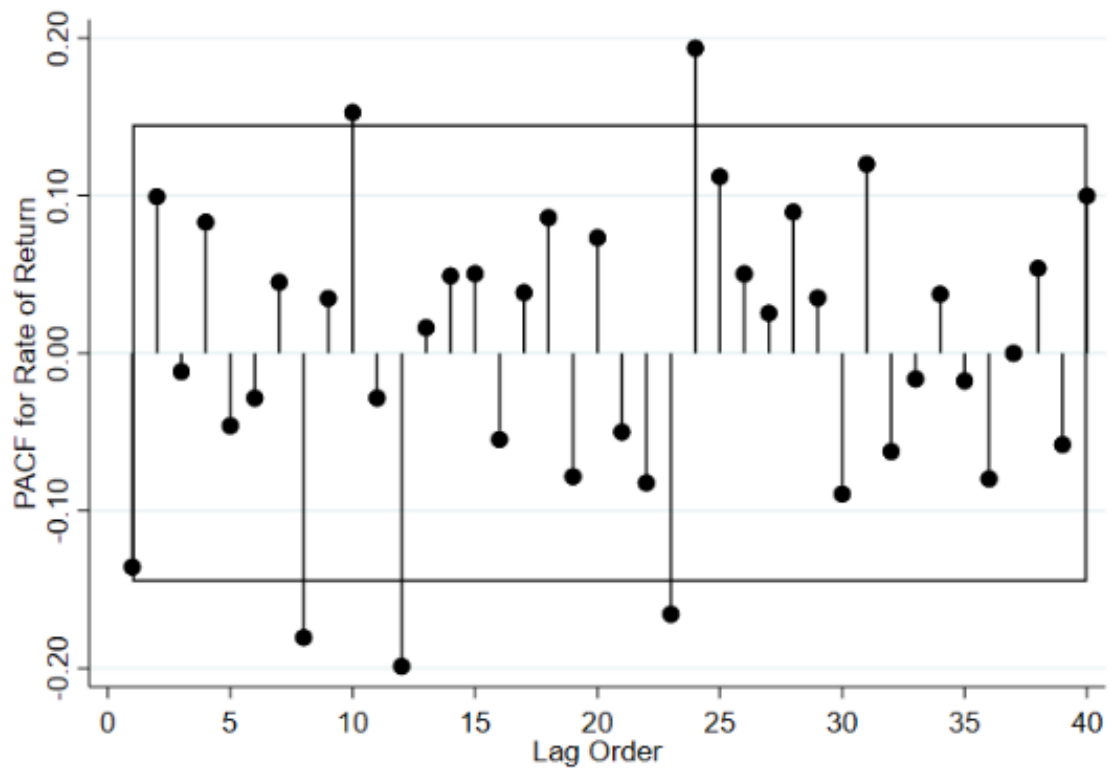

$95 \%$ Confidence bands $[$ se $=1 /$ sqrt $(n)]$

Figure 3. The time series asset of Auto correlation Function (ACF).

For PACF, instead of finding the present value, it aims to find the correlations left after removing the effects as the residuals.

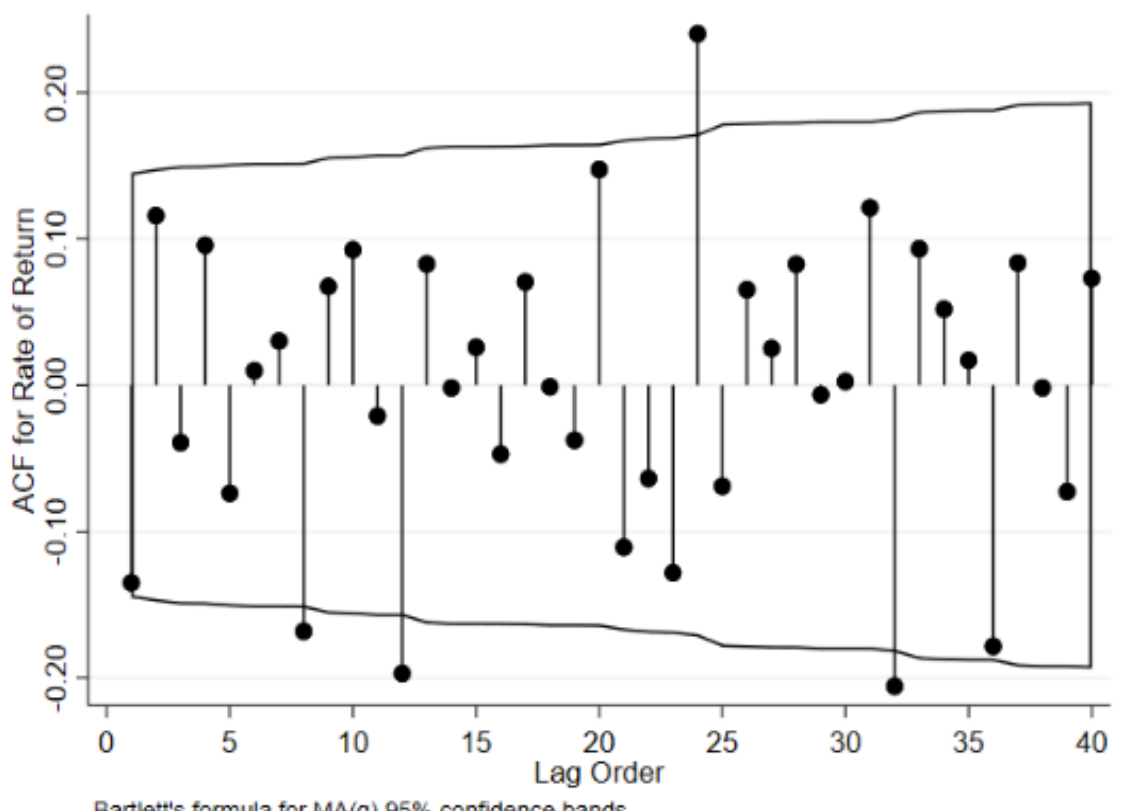

Bartlett's formula for MA(q) $95 \%$ confidence bands

Figure 4. The time series asset of Partial Autocorrelation Function (PACF).

To conclude ACF and PACF, we use ACF plot for finding optimum order of 'MA', and we use PACF plot for finding optimum order of 'AR'.

In Figure 3. and Figure 4., both graphs are bounded by a blush dash line, and the horizontal part of this (covering both positive and negative value) represents the significant threshold and only the spike that exceeds these two lines are considered as significant. As we can tell that both graphs are tails off, the ARMA model needs to be used. We may not deduce the order of $(p, q)$ from the plot, hence $\mathrm{R}$ studio is needed. 


\subsubsection{The Use of ARMA Model}

After deciding which model to use, we may be able to find the $(p, q)$ value with the help of $\mathrm{R}$ studio. And the auto ARMA value is $(8,8)$.

\subsubsection{Setting Dummy Variables}

We are setting three dummy variables in this research, and we will name them 'd1',' d2', and 'd3'. And the value range is based on the period, considering the policies given by the China Internet Finance Association and the other two organizations on May 18, 2021.

d1 is considered a period of a week, and it starts from May 18, 2021, to May 24, 2021; the value in this period we take as 1 , otherwise is 0 .

$\mathrm{d} 2$ is considered a period of two weeks, and it starts from May 18, 2021, to May 31, 2021; the value in this period we take as 1 , otherwise is 0 .

d3 is considered three months, and it starts from May 18, 2021, to the end of the asset date; the value in this period we take as 1 , otherwise is 0 .

\subsubsection{GARCH (Auto-Regressive Conditional}

\section{Heteroskedasticity) Model}

By using the GARCH model [7], it is shown in the way in which volatility can change. The lag length $(p)$ of the $(p, q)$ process is established steps shown below.

First, let us rewrite the 'AR' equation by sub into $q=8$, and the equation is demonstrated below [8].

$$
y_{t}=\mu+\sum_{i=1}^{8} \phi_{i} y_{t-i}+\epsilon_{t}
$$

Secondly, we need to compute and plot the autoauto-correlation with the following equation.

$$
\rho=\frac{\sum_{t=i+1}^{T}\left(\hat{\epsilon}_{t}^{2}-\hat{\sigma}_{t}^{2}\right)\left(\hat{\epsilon}_{t-1}^{2}-\hat{\sigma}_{t-1}^{2}\right)}{\sum_{t=1}^{T}\left(\hat{\epsilon}_{t}^{2}-\hat{\sigma}_{t}^{2}\right)^{2}}
$$

Last, we will use the following three formulas to demonstrate our data asset.

$$
\begin{gathered}
y_{t}=x_{t}^{\prime} b+\epsilon_{t} \\
\epsilon_{t} \mid \psi_{t-1} \sim N\left(0, \sigma_{t}^{2}\right) \\
\sigma_{t}^{2}=\omega+\sum_{i=1}^{q} \alpha_{1} \epsilon_{t-1}^{2}+\sum_{i=1}^{p} \beta_{1} \sigma_{t-1}^{2}
\end{gathered}
$$

In this case, $p$ is the order of the GARCH terms $\sigma^{2}$; and $q$ the order of the ARCH terms $\epsilon^{2}$.

And if we are rewriting (10), we shall get what is showing below.

$$
\sigma_{t}^{2}=\omega+\sum_{i=1}^{8} \alpha_{1} \epsilon_{t-1}^{2}+\sum_{i=1}^{8} \beta_{1} \sigma_{t-1}^{2}
$$

\section{EMPIRICAL RESULTS}

In this section, we list down the logarithmic returns rate of its original value and adjusted value; and compare and analyze the data. Coefficient values and standard error have been written down for each part. 182 data is observed in this research.

\subsection{Logarithmic Returns Rate Table}

Table 1. Historical performance of the mean equation of logarithm returns rate in the 6 months (the adjusted closing value); all the data are calculated daily.

Adjusted logarithmic returns rate

Mean Equation

(1) (2)

$\begin{array}{lllll}\text { L8.AR } & \text { Coef. } & -0.0210 & -0.0209 & -0.1837 \\ & \text { Std.Err. } & 0.2529 & 0.2453 & 0.2403 \\ \text { L8.MA } & \text { Coef. } & -0.0796 & -0.0828 & -0.0856 \\ & \text { Std.Err. } & 0.2238 & 0.2178 & 0.2147\end{array}$

number of observations: 182

Table 2. Historical performance of the variance equation of logarithm returns rate in the 6 months (the adjusted closing value); all the data are calculated in a daily period.

Adjusted logarithmic returns rate

Variance Equation

(1) (2)

D1

Coef.

1.7886

1.6635

1.6476

Std.Err.

0.3445

0.4020

0.4153

D2

Coef.

0.1561

0.2006

Std.Err.

0.3367

0.3995

D3

Coef.

$-0.0763$

Std.Err

0.2602

$\mathrm{ARCH}$

Coef.

$-0.0630$

$-0.0620$

$-0.0649$

Std.Err.

0.0185

0.0191

0.0222

GARCH COEF. $\quad-0.0815 \quad-0.1154 \quad-0.1151$

Std.Err. $\quad 0.0493 \quad 0.0877$ 


\begin{tabular}{lllll} 
Cons & Coef. & 0.00001 & 0.00005 & 0.00012 \\
& Std.Err. & 0.00295 & 0.00292 & 0.00294 \\
& & \multicolumn{4}{c}{ number of observations: 182}
\end{tabular}

In Table 1. and Table 2., 'Coef' stands for coefficients, and 'Std.Err' stands for standard error.

In order to restrain speculations and prevent sharp fluctuations in price, relevant policies have been issued in China in the way of Macro supervision. China Internet Finance Association and other organizations have jointly issued the "Notice on prevention of bitcoin risk" and "Announcement on preventing the financing risk of token issuance" on May 18, 2021. This will guard against the risk of speculation in virtual currency transactions. Four provisions are included, and the aim is to help the public investors give a correct understanding of the essential attributes of virtual currency and related business activities for first; which helps the investors calm down before investment. Secondly, to set up some limitations on relevant institutions, they shall not carry out business related to virtual currency. Furthermore, consumers should improve their awareness of risk prevention and beware of their property and rights of losses. Last but not the least, is to strengthen their self-discipline management of member units.

Table 3. Significant level for D1, D2 and D3.

Adjusted logarithmic returns rate

Variance Equation

$\begin{array}{ccccc}\text { D1 } & \mathrm{p} & 0 & 0 & 0 \\ \mathrm{D} 2 & \mathrm{p} & & 0.670 & 0.616 \\ \mathrm{D} 3 & \mathrm{p} & & & 0.769\end{array}$

number of observations: 182

From Table 3., looking at the coefficient of 'D1', we may find out that it presents significantly at the $1 \%$ significant level, which means in the period of 'D1', fluctuation occurs in the price of the bitcoin market. Hence marked effect is present after policies have been released for the first week.

For 'D2' and 'D3', we can easily find that the value is not significant compared to the value in 'D1'. This shows us that this impact is only for short terms, and the influence may be diluted by time in the electronic cryptocurrency Market.
With the policies, it gives a warning to most consumers in the short-term, especially to the one who is used to be fashion-follow consumers. From the data, we may find out that those policies do warn them but may not stop them from purchasing in the long run.

\subsection{Real-life results on price of bitcoin}

From the data, we may not be able to clear out that policies are causing great influence on the long run term of bitcoin price, but the fact is that the shrink on price is quite sharp to capture.

Since the policies have been released, austerity cracking down on bitcoin mining can be seen in many China areas (examples like Inner Mongolia, Sichuan, and Xinjiang). For some historical reasons, China has always been a quite popular center of 'mining' bitcoin; the Macro-regulation seems like coming in the way of unpredictable making plenty of mine's shutdown. In this way, a whopping drop in the price of bitcoin occurs. According to the research, the computing power of bitcoin reached a historical high of 176.06EH/S(on May $15,2021)$ and then goes straightly deceleration to 99.38EH/S(on July 15, 2021); and that is only two months, it has caused a decline more than $43.55 \%$ [9].

In July this year, Bitland announced the cooperation with Enegix to establish the world's largest encryption mine in Kazakhstan, which would be equipped with more than 50000 ant S19 Pro mining machines; this move eased the continued collapse of bitcoin price and extending the market out of China [10].

\section{CONCLUSION}

In this experiment, we used the ARMA-GARCH model to analyze the change in the price of bitcoin. From the data, we may conclude that macro-regulation does give control on the price of bitcoin directly in the short term, but there is also an indirect connection between them causing by the chain reaction.

After the research, we find that even with the restrictions causing shut down of those mines causing the price to decrease, people may also find their new ways in investing or moving mine's location outside the country (and push the price of bitcoin accelerating up in a gentle speed). Hence, the strike on purchasing bitcoin is not absolute but relative; it will be quite useful to lead novice buyers by considering them carefully before investing.

Our research proves that macro-regulation does negatively influence the price of bitcoin, and it might not be a permanent control, but it is still worth trying as it benefits in protecting the environment.

Unfortunately, there are still some problems with our research as we do not consider the close price; if it 
is included, a clear demonstration of how adjusted factors affect the price will be easy to compare.

\section{REFERENCES}

[1] Jason, P; Kelsie, N. Reason for bitcoin to have an all time high price, and how it value determinate. https://theconversation.com/why-is-bitcoins-priceat-an-all-time-high-and-how-is-its-valuedetermined-152616.

[2] Sebastian, S. Bitcoin Suffers Steepest Drop by Macro-regulation polices in United States. https://www.coindesk.com/markets/2021/06/08/bit coin-suffers-steepest-drop-in-10-days-as-usmonetary-policy-causes-short-term-jitters/.

[3] Bitcoin adjusted price download from yahoo finance. https://finance.yahoo.com/quote/BTCUSD/history? $\mathrm{p}=\mathrm{BTC}-\mathrm{USD}$.

[4] China Internet Finance Association and other two organizations have jointly issued the 4 restriction policies on the transaction of bitcoin. http://www.cs.com.cn/xwzx/hg/202105/t20210518 6168004.html.

[5] Benefits of using adjusted closing price in this research.

https://www.investopedia.com/terms/a/adjusted_cl osing_price.asp.

[6] Robert, H. Benefits of using logarithm returns rate in the measurement. http://papers.ssrn.com/sol3/papers.cfm?abstract_id $=1549328$.

[7] Erika, R. Brief usage of GARCH model in economic. https://www.investopedia.com/terms/g/garch.asp.

[8] Liang, S. Example of using ARIMA-GARCH model to predict the stock price after 10 working days. https://kns.cnki.net/KXReader/Detail?TIMESTAM $\mathrm{P}=637680089804195057 \& \mathrm{DBCODE}=\mathrm{CJFD} \& \mathrm{TAB}$ LEName $=$ CJFDLAST2020\&FileName $=$ KJHU202 012029\&RESULT $=1 \&$ SIGN=VuuonDZ1ufpkEH KM4e\%2fQ5Xi6\%2fWY\%3d

[9] Cihao, X. Huge decline in bitcoin price causing by shutting down of mines; due to the release of the four policies. https://www.163.com/dy/article/GITQ7UCF0550B 1DU.html.

[10] Jamie, C. Kazakhstan Data Center has Operator Enegix to Host Bitmain Machines on July. https://www.coindesk.com/markets/2021/07/23/kaz akhstan-data-center-operator-enegix-to-hostbitmain-machines/. 